\title{
Visual EXPERIENCES Without \\ Presentational Phenomenology
}

\author{
KENGO MIYAZONO \\ Hokkaido University
}

\begin{abstract}
A number of philosophers claim that visual experiences have a peculiar phenomenal character that is "presentational". According to what I call the "Visual Presentationality Thesis", this peculiar phenomenal character, presentational phenomenology, is not merely a contingent feature but is a necessary feature of visual experiences. Necessarily, visual experiences have presentational phenomenology. The main aim of this paper is to argue against the Visual Presentationality Thesis. I refute the Visual Presentationality Thesis by giving some counterexamples to it. In particular, I give counterexamples from derealization, which is a psychopathological condition that is characterized by the subjective impression of unreality or detachment from one's surroundings. Derealization is a condition in which patients have visual experiences without presentational phenomenology. I defend this hypothesis about derealization on the basis of an inference to the best explanation; this hypothesis provides the best explanation of (otherwise puzzling) subjective reports by patients with derealization.
\end{abstract}

\section{Introduction}

A number of philosophers claim that visual experiences have a peculiar phenomenal character that is "presentational". Sturgeon, for example, says that visual phenomenology will be "as if a scene is made manifest to you" or "as if a scene is simply presented" (2000: 9). Similarly, Foster claims that visual appearance gives the subjective impression "of being directly presentational" or, in other words, the impression that an external object "is before his mind" in a "qualitatively transparent and ontologically immediate way" (2000: 50).

Contact: Kengo Miyazono <miyazono@let.hokudai.ac.jp> 
Let us call this phenomenal character "presentational phenomenology" (Chudnoff 2012; 2013). Presentational phenomenology ("PP" hereafter) is a phenomenal feature of visual experiences; that is, PP concerns what it is like to have visual experiences. To say that a visual experience has $\mathrm{PP}$ is to say that what it is like to have the visual experience involves the impression that the object of the experience, $\mathrm{O}$, is experienced in the way described in the quotes above-that is, as if $\mathrm{O}$ and its properties are directly presented before the mind.

The idea of PP (or something similar) has become increasingly popular in recent years, in particular in the discussions of the phenomenal account of perceptual justification. Similar ideas can also be found in the continental phenomenological tradition, most notably in Husserl (2001) (see Section 2.2 for more on PP).

I agree with Sturgeon, Foster, and other philosophers that visual experiences have PP, at least in typical cases. But can we say something stronger than this? Can we say that PP is a distinctive feature of visual experiences? To say that PP is a distinctive feature of visual experiences is to endorse the following two claims:

(1) Necessarily, visual experiences have PP.

(2) Necessarily, mental states with PP are visual experiences.

The second claim can be challenged. One might think that both visual experiences and non-visual sensory experiences (such as auditory experiences) can have PP. Some philosophers point out the phenomenological similarities between perceptual experiences and intuitions; they argue that PP can be one of the common features between them. ${ }^{1}$

How about the first claim? Let us call it the "Visual Presentationality Thesis". The Visual Presentationality Thesis is a tempting idea; it is tempting to think that $\mathrm{PP}$ is not merely a contingent feature but is a necessary feature (or, in Macpherson's 2015 terminology, a "structural feature") of visual experiences. Some might find it difficult to conceive of visual experiences without PP. Sturgeon writes: "there is no way to conceive visual phenomenology apart from SceneImmediacy [= PP]. What it's like to enjoy visual experience is for it to be as if objects and their features are directly before the mind" (2000: 24). ${ }^{2}$

I am, however, skeptical about the Visual Presentationality Thesis, and the main aim of this paper is to argue against it. My strategy in arguing against it

1. See Chudnoff $(2012 ; 2013)$ and Bengson (2015). Chudnoff goes on to argue that-in addition to perceptual and intuitive experiences - introspective, recollective, and imaginative experiences can have PP too.

2. But, of course, there is a further question as to whether the inconceivability of a visual experience without PP can be good evidence for the impossibility of such an experience. See Macpherson (2015) for a related discussion on the conceivability and possibility of experiences. 
is to give some counterexamples to it. In particular, I will give counterexamples from derealization, which is a psychopathological condition that is characterized by the subjective impression of unreality or detachment from one's surroundings (see Section 3.1 for more details). ${ }^{3}$

After clarifying the Visual Presentationality Thesis (Section 2), I will defend the hypothesis that derealization is a condition in which patients have visual experiences without PP (Sections 3 and 4). I will defend this hypothesis, the "Missing PP Hypothesis", on the basis of an inference to the best explanation; the Missing PP Hypothesis provides the best explanation of (otherwise puzzling) subjective reports by patients with derealization.

\section{Preliminaries}

\subsection{The Visual Presentationality Thesis}

The aim of this paper is to falsify the Visual Presentationality Thesis by introducing counterexamples. This preliminary section will explain what this thesis amounts to and will clarify what is needed to falsify it. It turns out that there are many different interpretations of the thesis. My general strategy is to choose the interpretation that makes the thesis as promising as possible, so that my project of falsifying the thesis is philosophically significant.

The Visual Presentationality Thesis says that, necessarily, visual experiences have PP. I will now clarify the three key components in this claim; "visual experiences", "necessity", and "PP".

Visual Experience. The Visual Presentationality Thesis is a thesis about visual experiences, not about perceptual experiences in general. I will not discuss other perceptual modalities in this paper. ${ }^{4}$ Visual experiences are conscious experiences. "Type-1" blindsight (Weiskrantz 2009), where the subject has no conscious awareness of the stimuli in the blind field, does not count as a visual experience. ${ }^{5}$

3. This idea is not entirely original. Similar ideas have been expressed in the philosophical literature (Billon 2016; Dokic \& Martin 2012; 2017; Teng 2020) and in the empirical literature (e.g., Seth, Suzuki, \& Critchley 2012). Related ideas can also be found in the tradition of phenomenological psychopathology (Varga 2012), most notably in Jaspers's General Psychopathology (1997). Indeed, I take my proposal to be an updated version of Jaspers's view, which I will discuss below. What I offer in this paper is not a new idea but rather a careful argument for it.

4. But I am inclined to think that my argument will be applicable to other modalities as well. In fact, derealization can involve altered experiences in different modalities, and my account will be equally applicable to them.

5. See Macpherson (2015) for a discussion of whether "Type-2" blindsight, where the subject has some conscious awareness, counts as a visual experience. See also Philips (2021) for a discussion of the plausibility of the Type-1/Type-2 distinction. 
By “visual experiences", I mean visual experiences in the broad sense, including veridical, illusory, and hallucinatory experiences. The Visual Presentationality Thesis thus attributes PP to visual experiences in the broad sense: "veridical perception, illusion and hallucination seem to place objects and their features directly before the mind" (Sturgeon 2000: 9). ${ }^{6}$

Necessity. In its simplest form, the Visual Presentationality Thesis says that, necessarily, visual experiences have PP. This thesis can be interpreted in multiple ways, depending on the different interpretations of "necessity" and "subject". For instance, a strong reading would take the relevant necessity to be metaphysical necessity and the relevant subjects to be any metaphysically possible creatures.

\section{The Metaphysical Visual Presentationality Thesis}

Metaphysically necessarily, visual experiences of metaphysically possible creatures have PP.

According to the Metaphysical Visual Presentationality Thesis (Metaphysical VPT hereafter), even for God (assuming that God cannot violate metaphysical necessity and that God can have visual experiences), objects of visual experiences are experienced as if they are directly presented before the mind.

Perhaps the Metaphysical VPT is unnecessarily strong and controversial. There are weaker but still philosophically interesting interpretations. I borrow the following idea from Bayne's discussion of the unity of consciousness. Explicating what he calls the "Unity Thesis" (which, roughly, states that consciousness is necessarily unified), Bayne says:

I will argue that we never have disunified experiences. Not only do we retain a unified consciousness within normal everyday contexts, we retain this kind of unity even in the context of the most severe impairments of consciousness. The mechanisms underpinning consciousness function in such a way that the conscious states they generate always

6. But one might think that this version of the Visual Presentationality Thesis is too strong. For example, some philosophers adopt the view that hallucinations do not have any (perceptual) phenomenology; we only mistakenly take them to have (perceptual) phenomenal character (e.g., Fish 2009; Logue 2012). These philosophers might think that this version of the Visual Presentationality Thesis is simply false on the grounds that hallucinatory experiences do not have any (perceptual) phenomenology in the first place. I am not very sympathetic to this view of hallucination. But, setting it aside, there is nothing surprising in finding that hallucinatory experiences do not have PP if it turns out that they do not have any (perceptual) phenomenology in the first place. Counterexamples of this kind can easily be avoided by modifying the Visual Presentationality Thesis slightly, making it a conditional claim; necessarily, all visual experiences, if they have some (perceptual) phenomenology, have PP. 
occur as the unified components of a single phenomenal field. However, I do not claim that it is a conceptual or metaphysical truth that our conscious states are always unified; indeed, I do not even claim that the unity of consciousness is grounded in the law of nature. (2010: 17, italics original)

According to Bayne, the kind of necessity involved in the Unity Thesis is weaker than metaphysical necessity and conceptual necessity, and it is even weaker than nomological necessity (i.e., necessity according to the laws of nature). The Unity Thesis is therefore consistent with the possibility of disunified consciousness due to some surgical interventions or evolutionary developments in the future. The thesis is also consistent with the existence of some non-human species with disunified consciousness.

In short, the "necessity" in the Unity Thesis is understood as a kind of necessity according to some empirical neuropsychological facts about humans (I will call it "neuropsychological necessity"), and the relevant "subjects" in the thesis are humans or, more precisely, humans like us (viz., not humans with very different neuropsychological features due to some surgical interventions or evolutionary developments in the future).

I formulate the Visual Presentationality Thesis in an analogy with Bayne's Unity Thesis; "necessity" in the Visual Presentationality Thesis is taken to be neuropsychological necessity, and the subjects of "visual experiences" are taken to be humans. From this, we get the following:

\section{The Neuropsychological Visual Presentationality Thesis}

Neuropsychologically necessarily, visual experiences of humans have PP.

From now on, I will focus on the Neuropsychological Visual Presentationality Thesis (Neuropsychological VPT hereafter) and try to falsify it. Since the Neuropsychological VPT is weaker than the Metaphysical VPT (and other versions of the thesis in terms of conceptual necessity, nomological necessity, etc.), counterexamples to the former are also counterexamples to the latter (and other versions).

\subsection{Presentational Phenomenology}

By "presentational phenomenology", I refer to the phenomenal character that is described in the quotes by the philosophers mentioned above, such as Sturgeon and Foster. Roughly, to say that a mental state M has PP (with respect to an 
external object $\mathrm{O}$ ) is to say that what it is like to be in $\mathrm{M}$ involves the impression that $\mathrm{O}$ and its properties are directly presented before the mind. The phrase "an object and its properties are directly presented before the mind" is not anything more than a rough characterization. It is not obvious that we can do a better job than providing a rough characterization given the general difficulty in giving the precise verbal description of phenomenal characters. ${ }^{7}$

In the philosophical literature, different philosophers adopt different terminology; for example, "Scene-Immediacy" (Sturgeon 2000), "presentational feel" (Foster 2000), "presentational phenomenology" (Chudnoff 2012; 2013; Kriegel 2015), "presentational phenomenal character" (Siegel 2017), "phenomenological directness" (Millar 2014a; 2014b), "presentational force" (Smithies 2019), "phenomenal force" (Pryor 2000; Siegel \& Silins 2015), "forcefulness" (Huemer 2001), "assertiveness" (Tucker 2010), "feeling of presence" (Ferretti 2018; Matthen 2010), "feeling of reality" (Dokic \& Martin 2012; 2017), and "bodily presence" (Husserl 2001). ${ }^{8}$

It is far from obvious that these terms and phrases above refer to the same thing. It is not easy to tell whether they refer to the same thing in different ways or whether they talk about slightly different things. But there are two crucial issues that need to be clarified before moving forward. One issue is about the different aspects of PP, and the other is about the degree of PP.

Aspects of PP: PP has been treated as something simple and unitary by many philosophers. However, it is useful to distinguish two different aspects of PP (Millar 2014a; 2014b). Think about a visual experience of a red apple on the table. One aspect of PP (Millar calls it "object-directness") involves the impression that the apple and its redness are immediately presented before the mind. The other aspect of PP ("object-distinctness") involves the impression that the apple and its redness are distinct from the very experience of them.

Object-directness and object-distinctness can be dissociated from each other. As Millar points out, pressure phosphenes, which people experience when they apply physical pressure to their closed eyes, are object-direct; the experience of phosphenes are as direct as the experience of a red apple on the table. But, the experience of pressure phosphenes is not object-distinct; unlike the red apple on

7. Chudnoff offers the following analysis of PP: "what it is for an experience to have presentational phenomenology with respect to $\mathrm{p}$ is for it to both make it seem to you that $\mathrm{p}$ and make it seem as if this experience makes you aware of a truth-maker for $\mathrm{p}^{\prime \prime}$ (Chudnoff 2013: 37). This is certainly an interesting attempt to characterize PP. However, it is not obvious that Chudnoff's analysis is any more precise than my characterization. In fact, he does not offer any further discussion of what is meant by seeming "as if this experience makes you aware of a truth-maker for $\mathrm{p}$ ". This is, of course, not to challenge his analysis. My point is just that in the end his analysis might just be another rough characterization.

8. For Husserl on PP, see Berghofer (2018) and Chudnoff (2012). 
the table, phosphenes are experienced as if they are constitutive aspects of the very experience of them.

The target of my discussion is object-directness rather than objectdistinctness. My hypothesis is that object-directness is lost in derealization. Derealization involves the loss of the impression that objects and their properties are immediately presented before the mind. I do not claim that derealization involves the loss of object-distinctness. In fact, it is likely that objects and their properties are felt to be distinct from the very experience of them even in the cases of derealization. Thus, there is a sense in which the loss of PP in derealization is a partial one.

If object-distinctness is intact in derealization, then derealization would be the mirror-image of pressure phosphene; derealization involves object-distinctness without object-directness, while pressure phosphene involves object-directness without object-distinctness. This implies that object-directness and objectdistinctness are doubly-dissociable; the former can exist without the latter, and the latter can exist without the former.

To simplify my discussion, I will not repeat the distinction between objectdirectness and object-distinctness in the rest of this paper. But this distinction is always in the background.

Degree of PP: My claim is that derealization involves visual experiences without PP, which are counterexamples to the Neuropsychological VPT. In making this claim, I am assuming the binary conception of PP, according to which either a mental state has PP or it does not.

However, some might challenge the binary conception. What if PP is graded in fact such that different visual experiences can have different degrees of PP? In fact, some philosophers are explicitly committed to the graded notion of PP. ${ }^{9}$

I do not rule out the graded conception of PP. However, if the graded conception is correct then I will need to revise my claims slightly. First, if the graded conception is correct, I will not claim that the degree of PP in visual experiences in derealization is zero. In fact, this claim is very hard to prove. I do not know how to show convincingly that the degree of PP in a particular case is zero rather than only very small. Instead, I claim that the degree of PP in visual experiences in derealization is abnormally low (and possibly zero).

Second, I will not claim that derealization provides counterexamples to the Neuropsychological VPT, which is formulated in terms of the binary conception

9. See, for example, the following quotes: "the phenomenological character of assertiveness comes in degrees. Some seemings are very weak, i.e., they are not very assertive. Other seemings are so assertive that they make their contents feel utterly obvious" (Tucker 2010: 530); "presentational force comes in degrees of 'perceptual confidence', which determine the degree of justification provided by perceptual experience" (Smithies 2019: 91). 
of PP. Instead I will say that derealization provides counterexamples to the following:

\section{The Neuropsychological VPT*}

Neuropsychologically necessarily, all visual experiences of humans have a normal degree of PP.

By "a normal degree" of PP, I mean a degree that is at least as large as the degree of PP in a typical quote from a philosopher (e.g., Sturgeon or Foster).

To simplify my discussion, I will adopt the binary conception throughout this paper. But my claims and arguments can also be reformulated in terms of the graded conception; for example, my phrase "a visual experience without PP" can be reformulated as "a visual experience with an abnormally low degree of PP".

\section{PP and Derealization}

\subsection{The Missing PP Hypothesis}

Now let us turn to the main part of this paper in which I submit the counterexamples to the Neuropsychological VPT. The counterexamples are found in derealization. Derealization might not be the only source of counterexamples, but I do believe that it is the most promising candidate.

In the following, I will first summarize some basic facts about derealization and then defend a hypothesis according to which derealization involves visual experiences without PP.

Derealization is often seen as a symptom of depersonalization syndrome. ${ }^{10}$ The term "depersonalization" can be confusing. It can refer to the whole syndrome (Sierra 2009), which includes derealization as one of its symptoms. It can also refer to a particular symptom, which is contrasted with derealization. To avoid any ambiguity, I use "depersonalization syndrome" to refer to the former and "depersonalization" to refer to the latter.

Derealization is characterized by the subjective impression of unreality or detachment from one's surroundings, and is often contrasted with depersonalization, which is characterized by the subjective impression of unreality or detachment from one's own mind, self, or body (although it is not obvious that a sharp distinction can be drawn between the two; see Sierra 2009).

10. "Depersonzalization/Derealization Disorder" in DSM-5; “Depersonalization-Derealization Syndrome" in ICD-10. 
Jaspers calls derealization "alienation from the perceptual world" and says that it is characterized by "odd, peculiar, eerie" (1997: 62) perceptual experiences. He insists that it is "a disturbance in the actual process of perception, not in its material elements nor in the apprehension of meaning nor in judgment" (1997: 63).

According to DSM-5, derealization "is characterized by a feeling of unreality or detachment from, or unfamiliarity with, the world, be it individuals, inanimate objects, or all surroundings", and the patient "may feel as if he or she were in a fog, dream, or bubble, or as if there were a veil or a glass wall between the individual and world around" (American Psychiatric Association 2013: 303).

Patients with derealization make distinctively peculiar subjective reports of their experiences:

Everything appears as through a veil [...] Things do not look as before, they are somehow altered, they seem strange, two-dimensional. [. . .] Everything seems extraordinary new as if I had not seen it for a long time. (Jaspers 1997: 62)

Looking at familiar things during a bad episode upsets me a lot. I look at them, but they don't seem real, they don't look the same and they don't look familiar anymore, even though I know deep down they are, I'm seeing things differently from how I used to, almost like I'm looking at something I know, but it doesn't feel like I know it any more. It feels like I'm looking through someone else's eye. (Sierra 2009: 27)

Familiar things look strange and foreign. [ . . ] It's all just there and it's all strange somehow. I see everything through a fog. Fluorescent lights intensify the horrible sensation and cast a deep veil over everything. I'm sealed in plastic wrap, closed off, almost deaf in the muted silence. It is as if the world were made of cellophane or glass. (Simeon \& Abugel 2006: 81)

Typical expressions in the subjective reports are nicely captured in the Cambridge Depersonalization Scale (Sierra \& Berrios 2000). Relevant items in the questionnaire include:

Out of the blue, I feel strange, as if I were not real or as if I were cut off from the world. (item 1)

What I see looks 'flat' or 'lifeless', as if I were looking at a picture. (item 2)

My surroundings feel detached or unreal, as if there were a veil between me and the outside world. (item 13) 
A remarkable feature of the subjective reports of derealization is that they are metaphorical, not literal. Patients use metaphors to express their altered experiences in derealization. Jaspers makes this explicit: "description always proceeds by metaphor as it is impossible to express the experiences directly. Patients do not think that the world has really changed but only feel as if everything were different to them" (Jaspers 1997: 62; see also Radovic \& Radovic 2002).

The metaphorical expressions in derealization are not random. There are some recurrent themes in them. Patients often say that they feel as if they were in a "fog", "dream", or "bubble", or as if there were a "veil" or a "glass wall" between them and external objects. Metaphors of this kind seem to express the idea of indirectness or detachment. Patients also say that they feel as if they were looking at a "picture" or a "movie", or as if external objects were "flat". Metaphors of this kind seem to express the idea of representation. ${ }^{11}$

I will now defend my hypothesis about derealization, which is stated as follows:

\section{The Missing PP Hypothesis}

The peculiar subjective reports of derealization are (at least partially) the metaphorical expressions of visual experiences without PP.

The Missing PP Hypothesis is not completely new. Jaspers expresses a similar idea: "in every normal perception there must be yet another factor which elude us had these patients not presented us with these peculiar complaints" (1997: 63). I agree with Jaspers, and I only add that the "another factor" is PP, and that it tends to elude us probably because it is so constant in our everyday perceptual lives that everybody (except for some careful philosophers) takes it for granted. ${ }^{12}$

11. One might think that the subjective reports of derealization express the idea of unfamiliarity; some objects in the environment look unfamiliar. This interpretation suggests the hypothesis that derealization is qualitatively similar to "delusional mood" (or "delusional atmosphere") in schizophrenia, where external objects look "unfamiliar" or "uncanny". This hypothesis deserves serious consideration (e.g., Sass, Pienkos, Nelson, \& Medford 2013), but there is at least a prima facie difficulty about it. The subjective reports of delusional mood do not usually contain the metaphors that characterize the subjective reports of derealization, such as "fog", "veil", or "picture". A possible interpretation of this difference is that there is an important phenomenological difference between derealization and delusional mood.

12. There can be an even deeper connection between the Missing PP Hypothesis and Jaspers's proposal. He does not discuss the nature of the "another factor" any further in his discussion of derealization. But in another place in General Psychopathology-where he discusses general features of perceptions and the distinction between perceptions and mental images - he argues that in perceptions (but not in mental images) "the objects stand bodily before us (as 'tangibly present', 'vividly felt', and 'appreceived' or 'with a quality of objectivity')" (Jaspers 1997: 60; italics original). It is natural to regard this bodily presence as the "another factor" that is normally present but 
The Missing PP Hypothesis does not necessarily say that the loss of PP explains all aspects of derealization. First, it is possible that derealization is a complex phenomenon which involves not just the loss of PP but also some other factors (such as cognitive, perceptual, or affective factors; see Section 4). Second, it is possible that derealization is a heterogeneous condition in which different people have different experiences. The Missing PP Hypothesis might not be applicable to all cases of derealization. Or, even if it is applicable to all cases of derealization, it is still possible that some other factors are more salient than the loss of PP at least in some cases.

Note that, even with these limitations, the Missing PP Hypothesis provides counterexamples to the Neuropsychological VPT. To falsify the Neuropsychological VPT, we do not have to be committed to stronger hypotheses, such as the hypothesis that derealization is a simple phenomenon in which the loss of PP is the only factor, or the hypothesis that derealization is a homogeneous phenomenon in which all patients experience the loss of PP.

\subsection{An Inference to the Best Explanation}

In arguing for the Missing PP Hypothesis, I appeal to an inference to the best explanation - that is, the best explanation of the subjective reports of derealization is provided by the Missing PP Hypothesis.

This argument has two parts: the Missing PP Hypothesis gives a plausible explanation of subjective reports (Part 1), and alternative hypotheses do not explain them as plausibly as the Missing PP Hypothesis (Part 2).

Let us begin with Part 1. The Missing PP Hypothesis nicely explains why people tend to use metaphorical expressions in describing their altered experiences. Non-philosophers do not have relevant technical concepts such as the concept of "presentational phenomenology". When PP is lost in their visual experiences, patients (who are not philosophers, at least in most cases) do not have appropriate concepts for expressing the loss in a direct, non-metaphorical manner. This is why they appeal to metaphors, which is the best thing they can do. ${ }^{13}$

The Missing PP Hypothesis does not just explain why patients tend to use metaphors, it also explains why they use particular metaphors with particular

missing in derealization. And, if Jaspers's "bodily presence" is another name for PP, which would be a fair interpretation, then Jaspers's view is essentially the Missing PP Hypothesis.

13. Here I am simply assuming that "presentational phenomenology" is not a metaphorical expression. Strictly speaking, however, this assumption can be challenged. I characterized PP by some vague expressions, such as "directly presented" or "before the mind", that could be taken as metaphorical. Thus, a more careful statement of my claim here would be that the best thing patients can do is to use the the non-technical metaphors such as "veil" or "fog", rather than technical metaphors such as "presentational phenomenology". 
themes, such as indirectness or representation. As we have already seen, PP involves the sense of directness (e.g., "the subjective impression of being directly presentational" in the quote from Foster) and the sense of presentation (e.g., "as if a scene is simply presented" in the quote from Sturgeon). ${ }^{14}$ Thus, metaphors with themes like indirectness or representation are suitable for the job of expressing the altered visual experiences without PP in which the sense of directness or presentation is lost. In short, the metaphors in the subjective reports of derealization are exactly what we would expect from someone with visual experiences without PP.

Thus, the Missing PP Hypothesis gives a plausible account of the subjective reports in derealization. This concludes Part 1 of my argument.

Before moving on, I would like to respond to one possible objection to my general strategy, which heavily relies on the subjective reports of derealization. This objection challenges the inference to the best explanation by questioning the trustworthiness of the explananda (i.e., the subjective reports). This objection does not deny that patients with derealization have some altered perceptual experiences, but it does deny that their subjective reports are a reliable guide to the nature of the altered experiences. Subjective reports are based upon introspection. But, according to this objection, we should be careful about the reliability of introspection of subjective experiences (e.g., Schwitzgebel 2008) and, moreover, we should be particularly skeptical about introspection of mentally disordered people whose introspective capacity might be particularly unreliable due to their mental dysfunctions.

Now, I do agree that we should not idealize subjective and introspective reports. We should not blindly accept everything patients say about their experiences. But I do not agree that we should be particularly skeptical about the subjective reports of derealization just because those reporting them are mentally disordered. This is an unwarranted generalization. Introspective capacity could be compromised in some psychiatric conditions, but it is far from obvious that derealization is such a condition.

Perhaps we should be especially skeptical about introspective reports of a (psychopathological) condition $X$ if people with $X$ make random subjective reports that do not cohere with one another, or if their subjective reports do not cohere with some other data concerning X (e.g., scientific data). But derealization does not satisfy either of the conditions. The subjective reports of derealization

14. This does not necessarily mean that indirect or representational realism about visual experience cannot account for PP. PP is a phenomenal feature of visual experience, while indirect or representational realism is a view about the structural feature of visual experience. This paper is neutral on whether the direct or presentational phenomenology (PP) is compatible with the indirect or representational structure. My point here is simply that PP involves the subjective sense of directness or presentationality, not that PP implies the direct or presentational structure. 
are not random at all; they tend to be about the common themes such as indirectness or representation. As we will see in the next section, subjective reports and scientific data do not conflict.

\section{Alternative Hypotheses}

\subsection{Cognitive, Perceptual, and Affective}

The rest of this paper will be devoted to Part 2 of my "inference to the best explanation" argument, in which I will examine alternative hypotheses. Among possible alternatives, I focus on what I take to be the most important ones: the Cognitive Hypothesis, the Perceptual Hypothesis, and the Affective Hypothesis.

Let us start by clarifying what is needed for a hypothesis to be a plausible alternative to the Missing PP Hypothesis.

The first condition for a successful alternative is obvious. A plausible alternative hypothesis needs to be "plausible". It needs to offer an explanation of subjective reports, and the explanation needs to be at least as plausible as the one offered by the Missing PP Hypothesis. Let us call this the "plausibility condition".

The second condition, which is even more obvious, is that a plausible alternative hypothesis needs to be distinct from the Missing PP Hypothesis. It needs to be a genuine alternative to the Missing PP Hypothesis rather than the same hypothesis that is described in another way. Let us call this the "distinctness condition".

As I noted above, the Missing PP Hypothesis does not say that the loss of $\mathrm{PP}$ is the only factor in derealization. Some other factors can be involved. Thus, a genuine alternative to the Missing PP Hypothesis says not only that another factor is involved in derealization but also that derealization is totally explained by another factor, and hence the loss of PP is explanatorily redundant.

A hypothesis is thus a plausible alternative to the Missing PP Hypothesis if and only if it satisfies both the plausibility condition and the distinctness condition.

We are now ready to discuss possible alternatives.

(1) Cognitive Hypothesis. According to one hypothesis, the derealization is explained by some cognitive factors, such as judgments or beliefs (e.g., a patient believes that there is a veil in front of her). The cognitive factors might influence one's visual experiences in a top-down manner (but the visual experiences still retain $\mathrm{PP}$ ).

The Cognitive Hypothesis has an obvious problem with respect to the plausibility condition. As Jaspers (1997) insists, patients with derealization do not seem to hold such unusual beliefs (e.g., the belief about a veil). Their judgments 
seem to be intact, even in severe cases of derealization. But I do not rule out the possibility that the experience of derealization causes some (delusional) beliefs in some cases. For example, the experience of depersonalization (or a severe version of it) could be the cause of the Cotard delusion (e.g., Billon 2016). Similarly, the experience of derealization (or a severe version of it) can cause some delusional beliefs too (e.g., Broome 2012). Still, it is clear that derealization does not always cause delusional beliefs, which is enough to discredit this hypothesis.

(2) Perceptual Hypothesis. Another hypothesis is that derealization is explained by perceptual factors. More precisely, it is explained by the perceptual factors that have nothing to do with (the loss of) PP. For example, derealization is explained by some illusions or hallucinations, such as the hallucinatory experience of a veil. The hallucinatory experience retains PP; for example, the veil and the objects behind it are experienced as if they are presented before the mind.

It is possible that the experience of derealization can be accompanied by some visual distortions such as illusions or hallucinations. In fact, depersonalization syndrome involves some forms of illusory experience in which, for examples, objects look bigger (macropsia), smaller (micropsia), etc. But the Perceptual Hypothesis says something stronger; derealization is totally explained by visual illusions or hallucinations and thus the loss of PP is explanatorily redundant.

The Perceptual Hypothesis does not satisfy the plausibility condition. There is a problem with the empirical and clinical credibility of this hypothesis. For example, Jaspers carefully considers the phenomenology of derealization ("alienation from the perceptual world"), and clearly distinguishes it from illusions and hallucinations ("false perceptions"). Unlike illusions or hallucinations, derealization is related to "another factor which would elude us had these patients not presented us with these peculiar complaints" (Jaspers 1997: 63). Most psychiatrists and researchers agree with Jaspers on distinguishing derealization from illusions or hallucinations. Although some versions of the Perceptual Hypothesis were proposed in the early 2oth century, they are not widely accepted today partly because of the lack of coherence with clinical observations and partly because of the lack of empirical support (Sierra 2009: Ch. 1).

Another problem is about explaining the metaphorical expressions of derealization. I do not necessarily deny that the Perceptual Hypothesis gives a coherent explanation of the metaphorical expressions, or at least some of them such as "veil" or "fog". But I do not think that the explanation by the Perceptual Hypothesis is as plausible as the explanation by the Missing PP Hypothesis.

A difficulty is that metaphorical expressions are not necessary to express illusions or hallucinations. For instance, a person experiencing a hallucination of a veil in front of her can simply say "I see a veil" or "I see something like a veil", 
rather than "I feel as if there were a veil in front of me". Why does she choose the latter, which is a somewhat unusual expression of hallucinatory experiences? ${ }^{15}$

I do not claim, however, that visual illusions and hallucinations are never expressed metaphorically. We might express illusions and hallucinations metaphorically in some cases, in particular when they are indistinct or elusive. For example, I might describe my indistinct or elusive experience of the Hermann grid illusion by saying that "I feel as if there were grey spots at the intersections of the grid". ${ }^{16}$ But it is not likely that the metaphorical description "I feel as if there were a veil in front of me" is the expression of an indistinct or elusive experience of a hallucinatory veil. Jaspers notes, "we should always remember that in reality they can see, hear, and feel sharply and distinctly" (1997: 62).

Moreover, there is a crucial difference between the metaphorical expressions of derealization and the metaphorical expressions of illusions or hallucinations. The metaphorical expressions of illusions or hallucination are possible, but they are dispensable. The Hermann grid illusion can be expressed metaphorically, but it does not have to be. (For example, I can just say "I am aware of some indistinct grey spots at the intersections".) In contrast, the metaphorical expressions of derealization are not only possible. Without metaphorical expressions, it is very difficult to express derealization; "description always proceeds by metaphor as it is impossible to express the experiences directly" (Jaspers 1997: 62). The Missing PP Hypothesis nicely explains why it is so difficult to express derealization without metaphorical expressions; most patients do not have non-metaphorical expressions (such as "presentational phenomenology") for describing the visual experience without PP.

(3) Affective Hypothesis. The next hypothesis is that derealization is explained by affective factors. This is by far the most important alternative hypothesis because of its strong empirical and clinical credibility.

It is important to note that derealization is not an isolated phenomenon; it occurs along with other symptoms, such as anomalous experiences of one's self and one's body (depersonalization); emotional numbing (de-affectualization); anomalies in subjective recall (de-ideation); and so on. The isolated occurrence of derealization is rare (Sierra 2009). This means that an explanation of derealization cannot be isolated from an explanation of depersonalization syndrome as a whole. The general consensus among researchers is that affective abnormalities are at the core of depersonalization syndrome (Sierra 2009; Sierra \& Berrios 1998).

15. In fact, the subjective reports of clinical hallucinations (such as the ones in Charles Bonnet syndrome) are often non-metaphorical. For the subjective reports of Charles Bonnet syndrome, see Sacks (2012: Ch. 1) as well as Mogk and Mogk (2003: Ch. 8).

16. This example was suggested by an anonymous referee. 
This strongly suggests that derealization, as a symptom of depersonalization syndrome, has something to do with affective abnormalities. There could be different versions of the Affective Hypothesis with different details concerning how exactly derealization is linked to affective abnormalities. I will now examine two important examples: the Visual Hypoemotionality Hypothesis (by Sierra and colleagues) and the Presence Deficit Hypothesis (by Seth and colleagues). ${ }^{17}$ My worry will be that the former fails to satisfy the plausibility condition, while the latter fails to satisfy the distinctness condition.

\subsection{Visual Hypoemotionality}

Let us begin with the Visual Hypoemotionality Hypothesis. Sierra and Berrios (1998) propose a neurobiological account of depersonalization syndrome according to which the syndrome is constituted by two factors: a profound inhibition of emotional responses and a state of increased alertness. They speculate that this combination is a hard-wired vestigial response that used to play some useful roles in evolutionary history. There were some recurrent situations in which emotionally-driven behavioral responses, such as the fight or flight response, were likely to lead to some undesirable evolutionary consequences. Our ancestors dealt with those situations by inhibiting emotional responses to avoid the undesirable consequences and, at the same time, boost alertness and attentiveness to track survival-related information.

The inhibition of emotional responses, which is the first factor in Sierra and Berrios's model, is coherent with the empirical and clinical evidence for hypoemotionality (i.e., a lack of or decrease in affective experiences) in depersonalization syndrome. According to Sierra (2009), derealization is a consequence of hypoemotionality. Sierra hypothesizes that in normal cases visual experiences are constantly "emotionally colored" (i.e., their phenomenology is constantly influenced by affective states). What happens in the case of derealization is that hypoemotionality disrupts the normal process of emotional coloring, resulting in visual experiences with unusual phenomenology.

In response to the Visual Hypoemotionality Hypothesis, I do not dispute, at least in this paper, Sierra and Berrios's model of depersonalization syndrome as a whole, nor their speculations about the evolutionary origin of it. Regardless of

17. Another example might be the Metacognitive Feeling Hypothesis (Dokic \& Martin 2012; 2017; Teng 2020). But this might not be a genuine example of the Affective Hypothesis; it is not obvious that metacognitive feeling is genuinely affective. See Dokic and Martin (2017) on this issue. In any case, the Metacognitive Feeling Hypothesis might not be an alternative to the Missing PP Hypothesis; it is rather another version of the same hypothesis with some a particular commitment on the nature of PP that PP has something to do with metacognitive processings. 
whether the model is correct, it is strongly supported that hypoemotionality is a crucial aspect (and perhaps the essential aspect) of depersonalization syndrome. Assuming that affective states can influence visual phenomenology in general (Siegel 2006), it is likely that hypoemotionality in depersonalization syndrome has abnormal consequences in visual phenomenology, which Sierra refers to as the "failures of emotional coloring". These abnormal consequences in visual phenomenology would constitute at least an aspect of derealization. I accept all the claims so far, but I am not yet convinced that the Visual Hypoemotionality Hypothesis satisfies the plausibility condition.

In defense of the Visual Hypoemotionality Hypothesis, Sierra draws our attention to the similarity between the subjective reports of derealization and those of visual hypoemotionality in other conditions. Here is a quote from a patient with visual hypoemotionality due to prosopagnosia:

I loved flowers so much before ... Their charm doesn't enter my mind any more. Looking at the landscape through the window, I see the hills, the trees, the colours, but all those things cannot convey their beauty to me ... Everything looks ordinary, indefinite. I feel indifferent about it. What I lack is feeling. (Habib 1986: 578)

Sierra argues that the similarity between the subjective reports of derealization and those of visual hypoemotionality suggests a common underlying deficit, that is, the failure of emotional coloring in perception.

Although I do not deny the similarities between derealization and visual hypoemotionality (in other conditions), I hesitate to accept Sierra's strong claim that that subjective reports of visual hypoemotionality (in other conditions) are often "indistinguishable from those typical of patients with derealization" (2009: 148). As I have already pointed out, what is remarkable about the subjective reports of derealization is the use of metaphors, such as "fog" or "picture", that convey the idea of indirectness or representation. By using these metaphors, patients with derealization seem to express the impression that objects are no longer directly presented to them. In contrast, these metaphors do not appear in the subjective report of visual hypoemotionality above. What is remarkable in the subjective report is the complaint about aesthetic aspects (e.g., "charm", "beauty", "color") of one's visual scene. The patient seems to express the impression that objects no longer have their normal aesthetic qualities. This is also evident in another subjective report of visual hypoemotionality by one of Sierra's patients:

There is also an emptiness to landscapes. I cannot appreciate them, I cannot grasp the beauty of nature. I lack a kind of lucidity; a lucidity in 
my vision that would normally allow me to appreciate it; its colours, the temperature of its colours so to speak. I cannot think of a right word to explain it. I just cannot enjoy that sense of beauty that nature brings. (Sierra 2009: 149)

Given these dissimilarities, it is not obvious that derealization is fully explained in terms of the failure of emotional coloring. Perhaps the failure of emotional coloring is part of the explanation, but it does not tell the whole story of derealization. The Visual Hypoemotionality Hypothesis needs to be supplemented by another idea, which is, possibly, the Missing PP Hypothesis.

However, there are several possible responses in defense of the Visual Hypoemotionality Hypothesis. (1) One might insist that derealization and visual hypoemotionality share the same phenomenology (or very similar phenomenologies at least); it is just that different people express the same experience in different ways. For some reason, patients with derealization are biased towards expressing their experiences with metaphors, while patients with visual hypoemotionality are biased towards expressing their experiences with aesthetic vocabulary. (2) Derealization and visual hypoemotionality are different only in degree, not in kind. For example, the failure of emotional coloring is more severe in the former than in the latter. This severity explains why the former tends to be expressed with peculiar metaphors; the failure of emotional coloring is so severe in the case of derealization that patients cannot express their experiences without metaphors. (3) Patients with visual hypoemotionality focus on aesthetic aspects of visual experiences not because of phenomenological differences between derealization and visual hypoemotionality but simply because of the contingent facts about the way in which the interviewer asked questions in their interviews. For example, the quote above (from Sierra 2009) is a response to the question by the interviewer "What about landscapes?", which is very likely to lead the conversation to aesthetic topics. (4) Visual hypoemotionality in itself is not enough to explain derealization, but it can be combined with another factor to form a hybrid account of derealization. For example, derealization might be explained by visual hypoemotionality together with some visual illusions or hallucinations. ${ }^{18}$

I do not rule out these possibilities. My point here is only that, compared to the Missing PP Hypothesis, the Visual Hypoemotionality Hypothesis has explanatory disadvantages with regard to the empirical plausibility of its assumptions. The Visual Hypoemotionality Hypothesis accounts for the subjective reports of derealization only with some additional assumptions that have not been empirically confirmed; such as (1) patients with derealization are biased towards expressing their experiences with metaphors, (2) the failure of emotional coloring

18. This possibility was raised by an anonymous referee. 
is more severe in the derealization than in visual hypoemotionality (in other conditions), (3) the subjective reports of the visual hypoemotionality would have been very different if the interviewer had not asked about the landscape, (4) if some patients suffer from visual hypoemotionality and some relevant illusions or hallucinations at the same time, their subjective reports will be the same as, or at least very similar to, the subjective reports of derealization, etc. In contrast, the Missing PP Hypothesis explains the subjective reports of derealization without additional assumptions that are not empirically confirmed.

Despite this problem, the core insight by Sierra and colleagues (i.e., the insight that derealization has something to do with affective abnormalities) might be salvaged if the hypothesis is suitably revised. Discussing such a revision is the task of the next section.

\subsection{Presence Deficit}

Seth, Suzuki, and Critchley (2012) propose another model that links depersonalization syndrome to affective abnormalities. According to their model, the relevant affective abnormalities in depersonalization syndrome consist in a reduced "sense of presence".

According to Seth and colleagues, "sense of presence" means "the subjective sense of reality of the world and of the self within the world" (2012: 1). The "sense of presence" is introduced demonstratively, as whatever it is that is reduced or lost in depersonalization syndrome. This means that what is meant by the "sense of presence" is determined by what is reduced or lost in depersonalization syndrome as a matter of fact. ${ }^{19}$

19. Seth and colleagues also associate the "sense of presence" with virtual reality. The "sense of presence" is something that is lost in depersonalization syndrome, and something that is artificially manipulated in virtual reality experience. It is certainly an interesting idea that what is lost in depersonalization syndrome is the same as what is manipulated in virtual reality experience. But more empirical studies and theoretical discussions are needed to justify this idea. I claim that PP is lost in derealization, but I am neutral on whether PP is manipulated in virtual reality. Many studies of "presence" in virtual reality rely on the questionnaires (e.g., Barfield \& Hendrix 1995; Lessiter, Freeman, Keogh, \& Davidoff 2001; Witmer \& Singer 1998), which tend to measure multiple factors. For example, the Presence Questionnaire by Witmer and Singer (1998) tracks multiple factors, such as control factors (e.g., "How responsive was the environment to actions that you initiated (or performed)?"), sensory factors (e.g., "How much did the visual aspects of the environment involve you?"), realism factors (e.g., "To what degree did you feel confused or disoriented at the beginning of breaks or at the end of the experimental session?"), and distraction factors (e.g., "How aware were you of events occurring in the real world around you?"). Careful studies and discussions are needed to examine, first, which factors in the questionnaires are related to PP and, second, how PP-related factors are influenced by virtual reality experience. See Martin and Dokic (2017) for a related discussion of presence and virtual reality. 
Seth and colleagues explain the "sense of presence" in terms of their "interoceptive predictive coding model of presence" (Seth, et al. 2012; see also Seth 2013; Seth \& Friston 2016; Gerrans 2019). This model is, roughly, a predictive coding version of the James-Lange theory of emotions, according to which emotions are grounded in an interoceptive of the physiological condition of the body. According to the interoceptive predictive coding model, the brain does not passively receive interoceptive signals in a bottom-up manner; rather it actively predicts interoceptive signals in a top-down manner and monitors the match or mismatch between the predicted and actual interoceptive signals. This process happens in many different, but connected, levels in the cortical hierarchy. The sense of presence "arises when informative interoceptive prediction signals are successfully matched to inputs so that prediction errors are suppressed" (Seth et al. 2012: 3).

Depersonalization syndrome, according to Seth and colleagues, is a presence deficit. It is characterized by a reduced sense of presence due to abnormalities in the suppression process, which is caused by pathologically imprecise interoceptive predictive signals.

I would like to add two clarificatory comments before examining this proposal.

First, although the interoceptive predictive coding model of presence belongs to the James-Lange tradition in psychology of emotion, the sense of presence should not be regarded as an "emotion" in a narrow sense. Seth and colleagues make it explicit that the sense of presence is not a specific episode in the stream of consciousness (such as a specific occurrence of an emotion); it is rather a structural feature of consciousness in general.

Second, the presence deficit is a general problem in depersonalization syndrome. Given its interoceptive origin, perhaps it has more to do with depersonalization, which involves the unreality of self, rather than derealization, which involves the unreality of external objects. In order to account for derealization, Seth and colleagues need a story about how depersonalization is translated into derealization. A promising option is to endorse an analogue of the Visual Hypoemotionality Hypothesis: that is, derealization is the result of a failure in the process in which visual experiences are colored by the sense of presence (rather than, or in addition to, particular emotions or moods). This idea assumes that the sense of presence (rather than, or in addition to, particular affective states) has constant influence on visual experiences in normal cases, resulting in a peculiar visual phenomenology that might be called the "visual sense of presence". What happens in derealization is that this constant influence is disturbed due to a presence deficit in depersonalization syndrome, resulting in the reduction or loss of the visual sense of presence.

The Presence Deficit Hypothesis, understood this way, is an updated version of the Visual Hypoemotionality Hypothesis in which the crucial factor is 
the abnormality concerning the sense of presence rather than the abnormality concerning particular emotions or moods.

Can the Presence Deficit Hypothesis be a plausible alternative to the Missing PP Hypothesis?

The Presence Deficit Hypothesis is certainly a possible explanation of derealization and is possibly just as plausible as the Missing PP Hypothesis. But I am not convinced that it should be understood as an alternative to the Missing PP Hypothesis. Is the hypothesis that visual sense of presence is reduced or lost in derealization different from the hypothesis that PP is reduced or lost in derealization? ${ }^{20}$ Is visual sense of presence different from PP?

It is theoretically tempting to identify the visual sense of presence (in the Presence Deficit Hypothesis) with PP (in the Missing PP Hypothesis). In this interpretation, what Seth and colleagues do is to provide a neuroscientific story underlying the Missing PP Hypothesis rather than to provide an alternative account of derealization. With their neuroscientific story, they help us to understand how and why PP is reduced or lost in the case of derealization. Generally, $\mathrm{PP}$ in visual experiences is the product of presence coloring. In normal cases, the successful suppression of informative interoceptive signals by top-down predictions constantly influence visual phenomenology, resulting in PP (which here equates to the visual sense of presence). What happens in derealization is that this constant influence is disturbed due to the presence deficit, resulting in abnormal visual experiences in which PP (viz., the visual sense of presence) is reduced or lost.

Linking the Presence Deficit Hypothesis to the Missing PP Hypothesis in this way is theoretically attractive because doing so enables us to tell a coherent account of derealization that is consistent with subjective reports as well as available empirical findings. On the one hand, the account is consistent with subjective reports that are infused with peculiar metaphorical expressions, which strongly suggest a lack of PP in derealization. On the other hand, the account is consistent with the available empirical findings from research on depersonalization syndrome, which strongly suggest affective abnormalities as the crucial factor of the syndrome.

Moreover, we might be able to explain the seeming phenomenological differences between derealization and visual hypoemotionality, which I mentioned above in the discussion of the Visual Hypoemotionality Hypothesis. For example, mere visual hypoemotionality involves the failure of emotional coloring, but not the failure of presence coloring. In contrast, derealization involves the failure of emotional coloring as well as the failure of presence coloring, which means

20. The idea of PP's being reduced, rather than lost, presupposes the graded conception of PP. See Section 2.2 for a relevant discussion. 
that visual experiences in derealization are doubly abnormal. This hypothesis explains some similarities between the two conditions (because of a common factor, i.e., the failure of emotional coloring) as well as important differences (because of a peculiar factor only in derealization, i.e., the failure of presence coloring).

Of course, this is nothing more than a speculation. Defending a full-fledged theory of PP on the basis of the predictive interoceptive theory of presence by Seth and colleagues requires filling-in various details and testing empirical predictions. I will not embark on such a project here; this paper is primarily concerned with ascertaining the presence or absence of PP in derealization, rather than with proposing a (neuroscientific or psychological) theory of PP. ${ }^{21}$

To sum up, there are different versions of the Affective Hypothesis. I discuss two notable examples: the Visual Hypoemotionality Hypothesis by Sierra and colleagues and the Presence Deficit Hypothesis by Seth and colleagues. The former might not be a plausible alternative to the Missing PP Hypothesis because it might fail to (fully) capture the phenomenology of derealization. The latter might not be a plausible alternative to the Missing PP Hypothesis because it might not be an alternative hypothesis at all.

\section{Conclusion}

My claim in this paper is that the Visual Presentationality Thesis (or, more precisely, the Neuropsychological VPT) is falsified by counterexamples in derealization. My argument for this claim takes the form of an inference to the best explanation. The best explanation of the subjective reports of derealization is given by the Missing PP Hypothesis, according to which derealization involves visual experiences without PP. This hypothesis nicely explains notable features of subjective reports (Part 1 of the argument), and it is not clear that there are any plausible alternatives (Part 2). Some hypotheses fail to explain subjective reports as plausibly as the Missing PP Hypothesis. Others fail to be alternatives to the Missing PP Hypothesis at all.

21. Interestingly, Seth (2014) discusses another notion, "perceptual presence", that is associated with another theory that he calls the "predictive perception account of sensorimotor contingencies". This theory is, roughly, a predictive coding version of the sensorimotor theory of perception (Noë 2004; O'Regan \& Noë 2001). Because of its tight connection with perception of external objects, the theory can also be considered as an account of PP. See Seth (2015) for a discussion of the relationship between his interoceptive predictive coding model of presence and the predictive perception account of sensorimotor contingencies. 
That being said, I admit that my argument (Part 2, in particular) is only tentative, partly because I haven't discussed all possible hypotheses, and partly because there will be more to be said about the hypotheses that I have discussed.

The main aim of this paper is to contribute to the philosophy of perception by revealing a fact about the phenomenology of visual experiences, namely the fact that visual experiences can lose PP. But my discussion can also contribute to psychiatry and psychopathology by offering an account of (the subjective experience of) derealization. Derealization is a particularly puzzling phenomenon, possibly even more puzzling than other psychiatric conditions, such as depression or schizophrenia, that are frequently discussed in philosophical, psychological, and psychiatric literature. Despite several attempts, ${ }^{22}$ it is not easy to make sense of the subjective reports of patients with derealization. The Missing PP Hypothesis, if true, advances our understanding of derealization; the puzzling subjective reports by patients are the (metaphorical) expression of visual experience without $\mathrm{PP}$.

\section{Acknowledgement}

I thank Lisa Bortolotti, Anna Ichino, Yukihiro Nobuhara, Maja Spener, Scott Sturgeon, Lu Teng, Genki Uemura, Uku Tooming, and anonymous referees for helpful comments and suggestions. Early versions of this paper were presented at University of Birmingham (October 2014), University of Tokyo (July 2016, October 2017), University College Cork (March 2017), University of Macau (November 2018), and National Yang-Ming University (September 2019). I acknowledge the support of JSPS KAKENHI (grant number 18Hoo605, 20Hoooo1, \& 21Hoo464).

\section{References}

American Psychiatric Association (2013). Diagnostic and Statistical Manual of Mental Disorders (5th ed.). American Psychiatric Publishing.

Barfield, Woodrow and Claudia Hendrix (1995). The Effect of Update Rate on the Sense of Presence within Virtual Environments. Virtual Reality, 1(1), 3-15.

Bayne, Tim (2010). The Unity of Consciousness. Oxford University Press.

Bengson, John (2015). The Intellectual Given. Mind, 124(495), 707-60.

Berghofer, Philipp (2018). Husserl's Conception of Experiential Justification: What It Is and Why It Matters. Husserl Studies, 34(2), 145-70.

22. For other philosophical discussions of depersonalization syndrome, see Billon (2016), Colombetti and Ratcliffe (2012), Gerrans (2019), Radovic and Radovic (2003), Ratcliffe (2009), and Varga (2012). 
Billon, Alexandre (2016). Making Sense of the Cotard Syndrome: Insights from the Study of Depersonalisation. Mind E Language, 31(3), 356-91.

Broome, Matthew (2012). Reality, Realness, and the Natural Attitude. Philosophy, Psychiatry, E Psychology, 19(2), 115-18.

Chudnoff, Elijah (2012). Presentational Phenomenology. In Sofia Miguens and Gerhard Preyer (Eds.), Consciousness and Subjectivity (51-729). Ontos Verlag.

Chudnoff, Elijah (2013). Intuition. Oxford University Press.

Colombetti, Giovanna and Matthew Ratcliffe (2012). Bodily Feeling in Depersonalization: A Phenomenological Account. Emotion Review, 4(2), 145-50.

Dokic, Jérôme and Jean-Rémy Martin (2012). Disjunctivism, Hallucinations, and Metacognition. Wiley Interdisciplinary Reviews: Cognitive Science, 3(5), 533-43.

Dokic, Jérôme and Jean-Rémy Martin (2017). Felt Reality and the Opacity of Perception. Topoi, 36(2), 299-309.

Ferretti, Gabriele (2018). Visual Feeling of Presence. Pacific Philosophical Quarterly, 99(51), 112-36.

Fish, William (2009). Perception, Hallucination, and Illusion. Oxford University Press.

Foster, John (2000). The Nature of Perception. Oxford University Press.

Gerrans, Philipp (2019). Depersonalization Disorder, Affective Processing and Predictive Coding. Review of Philosophy and Psychology, 10(2), 401-18.

Habib, Michel (1986). Visual Hypoemotionality and Prosopagnosia Associated with Right Temporal Lobe Isolation. Neuropsychologia, 24(4), 577-82.

Huemer, Michael (2001). Skepticism and the Veil of Perception. Rowman \& Littlefield.

Husserl, Edmund (2001). Logical Investigations (Vol. 2). J. L. Findley (Trans.). Dermot Moran (Ed.). Routledge.

Jaspers, Karl (1997). General Psychopathology (Vol. 1). J. Hoenig and Marian W. Hamilton (Trans.). Johns Hopkins University Press.

Kriegel, Uriah (2015). The Varieties of Consciousness. Oxford University Press.

Lessiter, Jane, Jonathan Freeman, Edmund Keogh, and Jules Davidoff (2001). A Cross-Media Presence Questionnaire: The ITC-Sense of Presence Inventory. Presence, 10(3), 282-97.

Logue, Heather (2012). What Should the Naïve Realist Say about Total Hallucinations? Philosophical Perspectives, 26, 173-99.

Macpherson, Fiona (2015). The Structure of Experience, the Nature of the Visual, and Type 2 Blindsight. Consciousness and Cognition, 32, 104-28.

Matthen, Mohan (2010). Two Visual Systems and the Feeling of Presence. In Nivedita Gangopadhyay, Michael Madary, and Finn Spicer (Eds.), Perception, Action, and Consciousness: Sensorimotor Dynamics and the Two Visual Systems (107-24). Oxford University Press.

Millar, Boyd (2014a). The Phenomenological Directness of Perceptual Experience. Philosophical Studies, 170(2), 235-53.

Millar, Boyd (2014b). The Phenomenological Problem of Perception. Philosophy and Phenomenological Research, 88(3), 625-54.

Mogk, Lylas G. and Marja Mogk (2003). Macular Degeneration: The Complete Guide to Saving and Maximizing Your Sight. Ballantine Books.

Noë, Alva (2004). Action in Perception. MIT press.

O’Regan, J. Kevin and Alva Noë (2001). A Sensorimotor Account of Vision and Visual Consciousness. Behavioral and Brain Sciences, 24(5), 939-73. 
Phillips, Ian (2021). Blindsight Is Qualitatively Degraded Conscious Vision. Psychological Review, 128(3), 558-584.

Pryor, James (2000). The Skeptic and the Dogmatist. Nô̂s, 34(4), 517-49.

Radovic, Filip and Susanna Radovic (2002). Feelings of Unreality: A Conceptual and Phenomenological Analysis of the Language of Depersonalization. Philosophy, Psychiatry, E Psychology, 9(3), 271-79.

Ratcliffe, Matthew (2009). Existential Feeling and Psychopathology. Philosophy, Psychiatry, \& Psychology, 16(2), 179-94.

Sacks, Oliver (2012). Hallucinations. Penguin Random House.

Sass, Louis, Elizabeth Pienkos, Barnaby Nelson, and Nick Medford (2013). Anomalous Self-Experience in Depersonalization and Schizophrenia: A Comparative Investigation. Consciousness and Cognition, 22(2), 430-41.

Schwitzgebel, Eric (2008). The Unreliability of Naive Introspection. Philosophical Review, $117(2), 245-73$.

Seth, Anil K. (2013). Interoceptive Inference, Emotion, and the Embodied Self. Trends in Cognitive Sciences, 17(11), 565-73.

Seth, Anil K. (2014). A Predictive Processing Theory of Sensorimotor Contingencies: Explaining the Puzzle of Perceptual Presence and Its Absence in Synesthesia. Cognitive Neuroscience, 5(2), 97-118.

Seth, Anil K. (2015). The Cybernetic Bayesian Brain: From Interoceptive Inference to Sensorimotor Contingencies. In Thomas Metzinger and Jennifer M. Windt (Eds.), Open MIND. MIND Group. https://doi.org/10.15502/9783958570108

Seth, Anil K. and Karl J. Friston (2016). Active Interoceptive Inference and the Emotional Brain. Philosophical Transactions of the Royal Society B: Biological Sciences, 371(1708), 20160007.

Seth, Anil K., Keisuke Suzuki, and Hugo D. Critchley (2012). An Interoceptive Predictive Coding Model of Conscious Presence. Frontiers in Psychology, 2(395), 1-16.

Siegel, Susanna (2006). Which Properties Are Represented in Perception? In Tamar S. Gendler and John Hawthorne (Eds.), Perceptual Experience (481-503). Oxford University Press.

Siegel, Susanna (2017). The Rationality of Perception. Oxford University Press.

Siegel, Susanna and Nicholas Silins (2015). The Epistemology of Perception. In Mohan Matthen (Ed.), The Oxford Handbook of the Philosophy of Perception (781-811). Oxford University Press.

Sierra, Mauricio (2009). Depersonalization: A New Look at A Neglected Syndrome. Cambridge University Press.

Sierra, Mauricio and German E. Berrios (1998). Depersonalization: Neurobiological Perspectives. Biological Psychiatry, 44(9), 898-908.

Sierra, Mauricio and German E. Berrios (2000). The Cambridge Depersonalisation Scale: A New Instrument for the Measurement of Depersonalisation. Psychiatry Research, 93(2), 153-64.

Simeon, Daphne and Jeffrey Abugel (2006). Feeling Unreal: Depersonalization Disorder and the Loss of the Self. Oxford University Press.

Smithies, Declan (2019). The Epistemic Role of Consciousness. Oxford University Press.

Sturgeon, Scott (2000). Matters of Mind: Consciousness, Reason and Nature. Routledge.

Teng, Lu (2018). The Epistemic Insignificance of the Feeling of Presence. Manuscript in preparation. 
576 - Kengo Miyazono

Tucker, Chris (2010). Why Open-Minded People Should Endorse Dogmatism. Philosophical Perspectives, 24, 529-45.

Varga, Somogy (2012). Depersonalization and the Sense of Realness. Philosophy, Psychiatry, \& Psychology, 19(2), 103-13.

Weiskrantz, Lawrence (2009). Blindsight: A Case Study Spanning 35 Years and New Developments. Oxford University Press.

Witmer, Bob G. and Michael J. Singer (1998). Measuring Presence in Virtual Environments: A Presence Questionnaire. Presence, 7(3), 225-40. 